\title{
High-sensitivity force measurement using entangled probes
}

\author{
Stefano Mancini and Paolo Tombesi \\ INFM, Dipartimento di Fisica, Università di Camerino, I-62032 Camerino, Italy
}

(November 11, 2018)

\begin{abstract}
We show the possibility to improve the measurement sensitivity of a weak force by using massive probes in an entangled state. This latter can be achieved by exploiting radiation pressure effects.

03.65.Ta, 42.50.Vk, 03.65.Ud
\end{abstract}

Typeset using REVTEX 
Since the original formulation of quantum mechanics, entanglement has been recognized as one of its most puzzling features [1,2]. During the last few years a considerable amount of literature focused on methods to prepare atoms in nonclassical and also entangled states appeared [3]. A striking achievement in this rapidly expanding field has been the recent entanglement of two gas samples of atoms [4]. Contemporarily we proposed to exploit the radiation pressure to entangle massive macroscopic oscillators, like pendular mirrors in a cavity [5]. Once the entanglement between macroscopic oscillators has been shown to be accessible, another important achievement would be to give a technological application of such a property of their states, by considering them as probes. Then, our aim is to show here the potentiality of using these entangled oscillators in quantum metrology. This was already recognized in Ref. [6], where it was proposed to use entangled atoms for very high precision clocks. We shall show, instead, that one can use entangled probes for very sensitive tiny forces measurements.

Essentially, a classical coherent force causes a displacement of the probe (e.g. a pendular mirror) resulting in the shift of its state in the phase space. Such a displacement would be recognized through a readout apparatus (e.g. radiation field) [7]. Therefore, the ultimate limit in this kind of measurement is represented by the width of the probe's probability density along the displacement direction. In the most performing case, the width of the oscillator's ground state probability density determines the so called Standard Quantum Limit (SQL) [7]. This state can be identified with the vacuum state at zero temperature, so, to beat it, one should put the probe at least in a squeezed state [8].

Cavities with one pendular mirror have already been studied [9], showing a variety of intriguing effects coming from optomechanical coupling [10]. Moreover, due to the recent technological developments in optomechanics, this area is now experimentally accessible [11]. Therefore, the aim of this paper is to show that the use of two entangled probes (two pendular mirrors) surely improves the sensitivity of a weak force measurement. 


\section{State preparation}

Let us consider a cavity with two (identical) pendular mirrors, playing the role of probes, whose free Hamiltonian can be written as

$$
\hat{H}_{0}=\hbar \Omega \sum_{j=1}^{2}\left(\frac{\hat{p}_{j}^{2}}{2}+\frac{\hat{q}_{j}^{2}}{2}\right),
$$

where $\hat{q}_{j}$ and $\hat{p}_{j}(j=1,2)$ are the (dimensionless) position and momentum operators (with commutation relation $\left.\left[\hat{q}_{j}, \hat{p}_{k}\right]=i \delta_{j k}\right)$ and $\Omega$ is their frequency.

In practical situations massive probes are always far from SQL [7], mainly due to the thermal noise associated to their (initial) state. Here, we shall show how to prepare a probes' state having a reduced uncertainty which improves their sensitivity. To this end we recast the model introduced in Ref. [5], where we have shown the possibility of entangling massive probes by exploiting the radiation pressure. We are going to consider a radiation field, the entangler, described by the cavity mode $\hat{b}$, mediating information between the two mirrors (probes). The interaction Hamiltonian, to be added to Eq.(1), results of the form

$$
\hat{H}_{\text {int }}=\hbar G \hat{b}^{\dagger} \hat{b}\left(\hat{q}_{1}-\hat{q}_{2}\right)
$$

where $G$ is the optomechanical coupling constant depending on the probes' mass [9]. Since we require an intense cavity field we are naturally lead to a quadratic form of $\hat{H}_{\text {int }}$ by the simple replacement $\hat{b} \rightarrow \beta+\hat{b}$, where now $\beta$ denotes the classical amplitude of the cavity field and $\hat{b}$ the quantum fluctuation. Then, we have the following linear Heisenberg equations

$$
\begin{aligned}
& \dot{\hat{b}}=i \Delta \hat{b}-i G \beta\left(\hat{q}_{1}-\hat{q}_{2}\right), \\
& \dot{\hat{q}}_{j}=\Omega \hat{p}_{j}, \\
& \dot{\hat{p}}_{j}=-\Omega \hat{q}_{j}+(-)^{j} 2 G\left(\beta^{*} \hat{b}+\beta \hat{b}^{\dagger}\right),
\end{aligned}
$$

where $j=1,2$ and $\Delta$ is the field detuning [5]. For a sufficiently large value of $\Delta$ the $\hat{b}$ mode can be adiabatically eliminated obtaining

$$
\begin{aligned}
& \dot{\hat{q}}_{j}=\Omega \hat{p}_{j} \\
& \dot{\hat{p}}_{j}=-\Omega \hat{q}_{j}+(-)^{j} \frac{(2 G|\beta|)^{2}}{\Delta}\left(\hat{q}_{1}-\hat{q}_{2}\right) .
\end{aligned}
$$


The solution of these equations reads

$$
\begin{aligned}
\hat{q}_{j}(t)= & \frac{1}{2}\left[\cos (\Omega t)+(-)^{j-1} \cos (\Theta t)\right] \hat{q}_{1}(0)+\frac{1}{2}\left[\sin (\Omega t)+(-)^{j-1} \frac{\Omega}{\Theta} \sin (\Theta t)\right] \hat{p}_{1}(0) \\
& +\frac{1}{2}\left[\cos (\Omega t)+(-)^{j} \cos (\Theta t)\right] \hat{q}_{2}(0)+\frac{1}{2}\left[\sin (\Omega t)+(-)^{j} \frac{\Omega}{\Theta} \sin (\Theta t)\right] \hat{p}_{2}(0),
\end{aligned}
$$

where we have introduced the quantity

$$
\Theta=\left\{\Omega\left[\Omega+2 \frac{(2 G|\beta|)^{2}}{\Delta}\right]\right\}^{1 / 2} .
$$

The corresponding expression for $\hat{p}_{j}(t)$ can be easily determined through the time derivative of the above Eq.(5).

Now, we evaluate the fluctuations over a separable thermal state as could be that of realistic massive probes. Such state can be written as

$$
\hat{\rho}_{t h}=\mathcal{Z} \exp \left[-\frac{\hat{H}_{0}}{k_{B} T}\right],
$$

where $\mathcal{Z}=\left[\sum_{n} \exp \left(-n \hbar \Omega / k_{B} T\right)\right]^{-2}$ with $k_{B}$ the Boltzmann constant and $T$ the equilibrium temperature. The average number of thermal excitations for each probe is $\mathcal{N}_{t h}=$ $\left[\operatorname{coth}\left(\hbar \Omega / 2 k_{B} T\right)+1\right] / 2[12]$. Then, it can be easily seen that if we turn off the entangler at time $t=\pi /(2 \Theta)$, we are left with the following variances

$$
\mathbf{C} \equiv \frac{1}{2}\left\langle\left(\hat{\mathbf{v}} \hat{\mathbf{v}}^{T}\right)+\left(\hat{\mathbf{v}} \hat{\mathbf{v}}^{T}\right)^{T}\right\rangle_{\hat{\rho}_{t h}}=\frac{1}{2}\left(\frac{1}{2}+\mathcal{N}_{t h}\right)\left(\begin{array}{cccc}
1+r^{-2} & 0 & 1-r^{-2} & 0 \\
0 & 1+r^{2} & 0 & 1-r^{2} \\
1-r^{-2} & 0 & 1+r^{-2} & 0 \\
0 & 1-r^{2} & 0 & 1+r^{2}
\end{array}\right),
$$

where $\hat{\mathbf{v}}^{T}=\left(\hat{q}_{1}, \hat{p}_{1}, \hat{q}_{2}, \hat{p}_{2}\right)$ and $r=\Theta / \Omega \geq 1$. By using Eq.(8) we recognize that for $r \gg 1$ it is possible to reduce the noise in the quadrature $\hat{q}_{1}-\hat{q}_{2}$ bringing the probes state close to an eigenstate of this quadrature. In such a case the probes' state would be a kind of EPR state [2]. To be more precise, a general separability criterion introduced in Ref. [5] gives

$$
\left\langle\left(\hat{q}_{1}-\hat{q}_{2}\right)^{2}\right\rangle_{\hat{\rho}_{t h}} \times\left\langle\left(\hat{p}_{1}+\hat{p}_{2}\right)^{2}\right\rangle_{\hat{\rho}_{t h}} \geq\left|\left\langle\left[\hat{q}_{j}, \hat{p}_{j}\right]\right\rangle_{\hat{\rho}_{t h}}\right|^{2},
$$


then, the condition $r^{2}>\left(1+2 \mathcal{N}_{t h}\right)$ guarantees entanglement. This leads to a great improvement in the minimum detectable force as we shall see.

Furthermore, after interaction (2), leaving the probes to freely evolve one ends up with a Gaussian state, say $\hat{\rho}_{12}$, characterized by the following covariance matrix

$$
\mathbf{C}(\phi)=\mathbf{R}(\phi) \mathbf{C R}^{T}(\phi), \quad \mathbf{R}(\phi)=\left(\begin{array}{cccc}
\cos \phi & \sin \phi & 0 & 0 \\
-\sin \phi & \cos \phi & 0 & 0 \\
0 & 0 & \cos \phi & \sin \phi \\
0 & 0 & -\sin \phi & \cos \phi
\end{array}\right),
$$

where $\phi$ represents the phase space rotation angle depending on the frequency $\Omega$ and on the elapsed time.

\section{Weak force measurement}

After state preparation, we consider the action of a classical force on the probes and its readout through radiation fields. Thus, the interaction Hamiltonian to be added to $H_{0}$ would now be

$$
H_{\text {int }}=-\hbar \Omega f\left(\hat{q}_{1}-\hat{q}_{2}\right)-\hbar g \hat{c}_{1}^{\dagger} c_{1} \hat{q}_{1}+\hbar g \hat{c}_{2}^{\dagger} c_{2} \hat{q}_{2}
$$

where $f$ is the dimensionless force strength. Here, the force is considered as acting on the

relative variables of the probes, as for example, it is the case of gravitational radiation [13]. Furthermore, $\hat{c}_{j}$ are operators describing the meter fields which deserve as readout. These could be proper modes of additional cavities. They are optomechanically coupled to the probes with a strength $g$.

Assuming again intense fields, we are led to linear Heisenberg equations

$$
\begin{aligned}
& \dot{\hat{c}}_{j}=i \Delta_{j} \hat{c}_{j}-(-)^{j} i g \gamma q_{j}, \\
& \dot{\hat{q}}_{j}=\Omega \hat{p}_{j}, \\
& \dot{\hat{p}}_{j}=-\Omega \hat{q}_{j}-(-)^{j} 2 g\left(\gamma^{*} \hat{c}_{j}+\gamma \hat{c}_{j}^{\dagger}\right)-(-)^{j} \Omega f,
\end{aligned}
$$


where $\gamma$ denotes the classical fields amplitude and $\hat{c}_{j}$ the quantum fluctuations. Tpically, it should be $|\gamma| \ll|\beta|$ since radiation pressure could blur the signal. That is also the reason why we now consider the entangler turned off.

Choosing $\Delta_{j}=0, \gamma \in \mathbf{R}$, and introducing the field quadratures $\hat{X}_{j}=\left(\hat{c}_{j}+\hat{c}_{j}^{\dagger}\right) / \sqrt{2}$, $\hat{Y}_{j}=-i\left(\hat{c}_{j}-\hat{c}_{j}^{\dagger}\right) / \sqrt{2}$, (with commutation relation $\left.\left[\hat{X}_{j}, \hat{Y}_{k}\right]=i \delta_{j k}\right)$, we immediately see that only the phase quadratures $\hat{Y}_{j}$ carrie out information about the force. As matter of fact, from Eqs.(12), we obtain

$$
\begin{aligned}
\hat{Y}_{j}(\tau) & =-(-)^{j} \frac{g \gamma}{\Omega} \sin (\Omega \tau) \hat{q}_{j}(0)-(-)^{j} \frac{g \gamma}{\Omega}[1-\cos (\Omega \tau)] \hat{p}_{j}(0) \\
& +2 \frac{(g \gamma)^{2}}{\Omega^{2}}[\Omega \tau-\sin (\Omega \tau)] \hat{X}_{j}(0)+\sqrt{2} \frac{g \gamma}{\Omega}[\Omega \tau-\sin (\Omega \tau)] f+\hat{Y}_{j}(0),
\end{aligned}
$$

where $\tau$ is the time duration of the force. Then, performing homodyne detection [14] on the meters fields one can get the following signal

$$
\left\langle\hat{Y}_{1}(\tau)+\hat{Y}_{2}(\tau)\right\rangle \equiv \mathcal{S}(\tau) f=2 \sqrt{2} \frac{g \gamma}{\Omega}[1+\Omega \tau-\cos (\Omega \tau)] f
$$

The corresponding noise can be calculated by means of the initial state of the probes, $\hat{\rho}_{12}$, and the vacuum noise for the meters. Thus, we get

$$
\begin{aligned}
\left\langle\left[\hat{Y}_{1}(\tau)+\hat{Y}_{2}(\tau)\right]^{2}\right\rangle & \equiv \mathcal{N}(\tau) \\
& =\left(\frac{g \gamma}{\Omega}\right)^{2} \sin ^{2}(\Omega \tau)\left[r^{-2} \cos ^{2} \phi+r^{2} \sin ^{2} \phi\right]\left(1+2 \mathcal{N}_{t h}\right) \\
& +\left(\frac{g \gamma}{\Omega}\right)^{2}[1-\cos (\Omega \tau)]^{2}\left[r^{-2} \sin ^{2} \phi+r^{2} \cos ^{2} \phi\right]\left(1+2 \mathcal{N}_{t h}\right) \\
& -2\left(\frac{g \gamma}{\Omega}\right)^{2} \sin (\Omega \tau)[1-\cos (\Omega \tau)]\left[r^{-2}-r^{2}\right] \sin \phi \cos \phi\left(1+2 \mathcal{N}_{t h}\right) \\
& +4\left(\frac{g \gamma}{\Omega}\right)^{4}[\Omega \tau-\sin (\Omega \tau)]^{2} \\
& +1
\end{aligned}
$$

The first three terms in Eq.(15) come from the probes state, while the fourth term is due to the back action of the meters modes (radiation pressure noise), and the final 1 is the shot noise term. It is worth noting that for $\Omega \tau$ integer multiple of $2 \pi$ the noise does no longer depend on the initial probes state [15], but for all other times it does. Such state in our 
case is characterized by $\mathcal{N}_{t h}, r$ and $\phi$. Assuming $\mathcal{N}_{t h}$ fixed, one might optimize $\phi$, and then reduce the noise by increasing $r$. In doing so we get

$$
\phi_{\text {opt }}=-\frac{1}{2} \arctan \left\{-2 \frac{\sin (\Omega \tau)[1-\cos (\Omega \tau)]}{\sin ^{2}(\Omega \tau)-[1-\cos (\Omega \tau)]^{2}}\right\}+n \frac{\pi}{2},
$$

where $n$ is an integer number.

The relevant quantity to study is the signal to noise ratio

$$
\mathcal{R}=\frac{|\mathcal{S}|}{\sqrt{\mathcal{N}}} f \geq 1, \quad \Longrightarrow \quad f_{\min }=\frac{\sqrt{\mathcal{N}}}{|\mathcal{S}|}
$$

which gives the minimum detectable force. The latter, for $\mathcal{N}_{t h}=0$ and $r=1$, represents the SQL.

In Fig. 1 we show $f_{\min }$ as function of the (scaled) time when the force is on. The improved sensitivity due to the probes correlations is especially evident for $\Omega \tau<2 \pi$. Above this limit, the meters back action noise becomes dominant, thus reducing the benefit. Such back-action noise could be reduced by decreasing the value of $(g \gamma / \Omega)$, i.e., the intensity of the meter fields, but this also affects the strength of the signal. Thus, it turns out that, for each $\tau$, there exist an optimum value of $(g \gamma / \Omega)$ as shown in Fig.2. Also notice in Figs.1 and 2 that the entanglement condition, $r^{2}>\left(1+2 \mathcal{N}_{t h}\right)$, allows to beat the SQL (bottom curves). With the present model, the sensitivity of the force measurement crucially depends on the value of $r$. The latter is related the optomechanical coupling constant $G$ and on the intensity of the entangler field $|\beta|^{2}$. For instance, the parameters values used in experiments of Ref. [11] permit to reach $r \approx 50$, which would allow to beat the SQL, even with $\mathcal{N}_{t h}=10^{3}$.

It is also to remark that the achievable improvements are not uniform in the phase space, since we have assumed the phase $\phi$ locked to the time duration $\tau$ of the force in Eq.(16). Thus, we have a kind of two-mode squeezed state, and the situation is analogous to the use of a single-mode squeezed state, where the direction of the reduced uncertainty should coincide with the one of the force displacement [8]. This enforces the conclusions of Ref. [16], about the equivalence of entanglement and squeeezing, while the more optimistic results of Ref. [17] are not applicable in this case. We would remark however that probes' entangled 
state seems experimentally accessible with present technology while probes' squeezed state does not.

Finally, since the entangler has been considered turned off prior the measurement, what will really limit the applicability of the above procedure would be the decoherence, which degrades the prepared meters state. Such a decoherence takes place in a time scale $\left(\Gamma \mathcal{N}_{t h}\right)^{-1}$ $[9,14]$ with $\Gamma^{-1}$ the mechanical relaxation time of the probes. Therefore, the time for rotation

$\phi$, together with the duration $\tau$ of the force, should be less than $\left(\Gamma \mathcal{N}_{t h}\right)^{-1}$. Thus, the model would be suitable for weak but impulsive forces [18]. Favorable conditions for its implementation could be found in Micro Electro-Mechanical Systems [19,20], or Atomic Force Microscopes [21], where large mechanical quality factors are achievable.

Summarizing, we have proposed high-sensitivity force measurement by exploiting nonclassical features of the probes, like entanglement. That would allow us to overcome the uncertainty related to the probes state and even to beat the SQL. Our scheme, even if oversimplified, may open new perspectives in quantum metrology [20], as well as in gravitational waves detection [13].

\section{ACKNOWLEDGMENTS}

This work has been supported by the INFM under the PAIS project "Entanglement and Decoherence". S. M. gratefully acknowledges financial support from Università di Camerino under the project "Giovani Ricercatori". 


\section{REFERENCES}

[1] E. Schrödinger, Naturwissenschaften 23, 807, 823, 844 (1935).

[2] A. Einstein, et al., Phys. Rev. 47, 777 (1935).

[3] J. I. Cirac, et al., Adv. At. Mol. Phys. 37, 237 (1996); D. J. Wineland, et al., J. Res. Natl. Inst. Stand. Technol. 103, 259 (1998); C. A. Sackett et al., Nature (London) 404, $256(2000)$.

[4] A. Kuzmich, et al., Phys. Rev. Lett. 85, 1594 (2000); B. Julsgaard, et al., Nature (London) 413, 400 (2001).

[5] S. Mancini, et al., Phys. Rev. Lett. 88, 120401 (2002).

[6] D. J. Wineland, et al., Phys. Rev. A 46, R6797 (1992). S. Huelga, et al., Phys. Rev. Lett. 71, 3865 (1997); V. Meyer, et al., Phys. Rev. Lett. 86, 5870 (2001).

[7] V.B. Braginsky and F.Ya. Khalili, Quantum Measuraments, Ed. by K.S. Thorne (Cambridge Univ. Press, Cambridge, 1992).

[8] J. N. Hollenhorst, Phys. Rev. D 19, 1669 (1979).

[9] A.F. Pace, et al., Phys. Rev. A 47, 3173 (1993); K. Jacobs, et al., Phys. Rev. A 49, 1961 (1994); S. Mancini and P. Tombesi, Phys. Rev. A 49, 4055 (1994); C. Fabre, et al., Phys. Rev. A 49, 1337 (1994); G. J. Milburn, et al., Phys. Rev. A 50, 5256 (1994).

[10] S. Mancini, et al., Phys. Rev. A 55, 3042 (1997). S. Bose, et al., Phys. Rev. A 56, 4175 (1997);

[11] I. Tittonen, et al., Phys. Rev. A 59, 1038 (1999); Y. Hadjar, et al., Europhys. Lett. 46, 545 (1999); P. F. Cohadon, et al., Phys. Rev. Lett. 83, 3174 (1999).

[12] V. Giovannetti and D. Vitali, Phys. Rev. A 63, 023812 (2001).

[13] A. Abramovici, et al., Science 256, 325 (1992); C. M. Caves, Phys. Rev. Lett. 45, 75 
(1980); R. Loudon, Phys. Rev. Lett. 47, 815 (1981).

[14] D. F. Walls and G. J. Milburn, Quantum Optics, (Springer, Berlin, 1995). To be rigorous, the homodyne measurement should be performed on the field outgoing a cavity. For the sake of simplicity we don't employ the input-output formalism. However, this does not affect the qualitative results.

[15] See also, V. B. Braginsky, et al., gr-qc/0109003, for a discussion on the dependence of the noise from the initial probes state.

[16] W. J. Munro, et al., quant-ph/0109049.

[17] G. M. D'Ariano, et al., Phys. Rev. Lett. 87, 270404 (2001).

[18] From this point of view, these results are somehow analogous with those obtained by using a feedback mechanism to prepare the initial state of a probe with improved sensitivity: D. Vitali, et al., Phys. Rev. A 64, 051401 (2001).

[19] W. S. Trimmer, Micromechanics and MEMS: Classical and Seminal Papers to 1990, (IEEE Press, New York, 1997).

[20] A.N. Cleland and M.L. Roukes, Appl. Phys. Lett. 69, 2653 (1996).

[21] J. Mertz, et al., Appl. Phys. Lett. 62, 2344 (1993); T. D. Stowe, et al., Appl. Phys. Lett. 71, 288 (1997). 


\section{FIGURES}

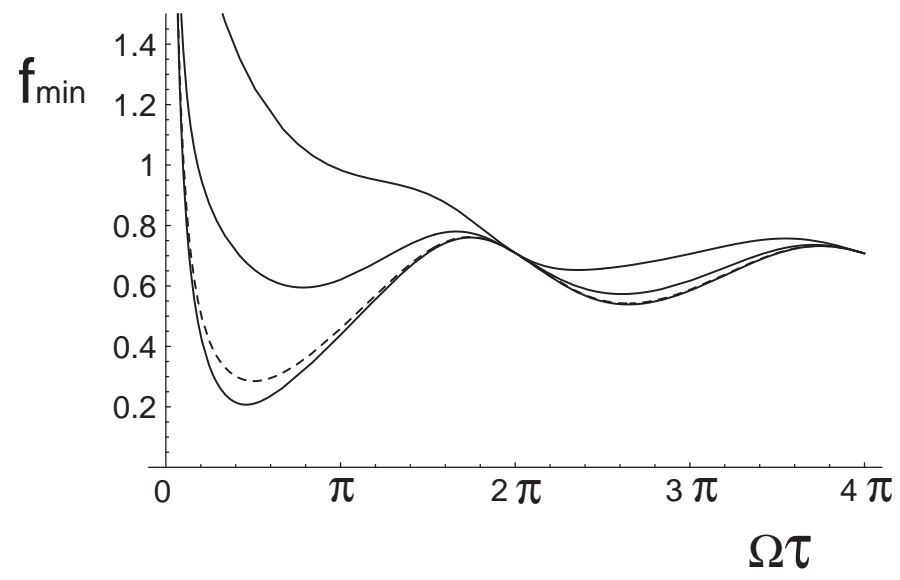

FIG. 1. The minimum detectable force $f_{\min }$ is plotted versus the scaled time $\Omega \tau$ for $\mathcal{N}_{t h}=20$ and $(g \gamma / \Omega)=1$. Solid curves from top to bottom are for $r=1, r=2$ and $r=10$. Instead, the dashed curve represents the SQL.

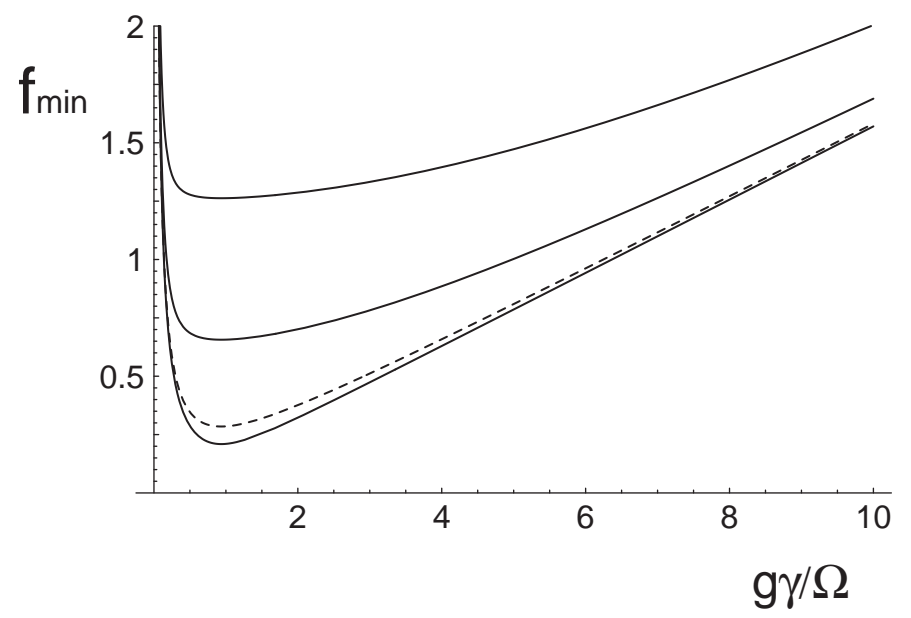

FIG. 2. The minimum detectable force $f_{\text {min }}$ is plotted versus the quantity $g \gamma / \Omega$ for $\mathcal{N}_{t h}=20$ and $\Omega \tau=\pi / 2$. Solid curves from top to bottom are for $r=1, r=2$ and $r=10$. Instead, the dashed curve represents the SQL. 